Pacific

Journal of

Mathematics

\title{
THE MEASURES OF ASYMMETRY FOR COPRODUCTS
} OF CONVEX BODIES

Qi Guo, JinFeng Guo And XunLi Su 


\title{
THE MEASURES OF ASYMMETRY FOR COPRODUCTS OF CONVEX BODIES
}

\author{
Qi GuO, JinFEng GuO AND XunLi Su
}

\begin{abstract}
In previous work, we introduced a family of $\boldsymbol{p}$-measures of asymmetry for convex bodies, which have the well-known Minkowski measure of asymmetry as a particular case. We now reveal more properties of 1-measure and $\infty$-measure and give some calculating formulas of $\boldsymbol{p}$-measures, in particular, for the so-called coproducts of convex bodies.
\end{abstract}

The measures of asymmetry for convex bodies, which in principle can be traced back to an early paper by Minkowski [1897], have been studied for a long time [Asplund et al. 1962; Besicovitch 1948; Chakerian and Stein 1964; Eggleston 1952; Klee 1953; Rogers and Shephard 1958; Stein 1956]. In particular, after B. Grünbaum formulated in his well-known paper [1963] a general definition of measures of (central) asymmetry (or symmetry), many mathematicians have contributed their efforts to this topic: studying the properties/applications of those known measures of asymmetry [Böröczky 2010; Dziechcińska-Halamoda and Szwiec 1985; Ekström 2000; Gluskin and Litvak 2008; Groemer 2000; Groemer and Wallen 2001; Guo 2005; Guo and Kaijser 1999; 2003; 2002; Hug and Schneider 2007; Kaiser 1996; Petitjean 2003; Schneider 2009; Soltan 2005; Mizushima 2000; Toth 2009; 2008], looking for new ones or studying other types of measure of asymmetry [Tuzikov et al. 2000; Tuzikov et al. 1997; Zouaki 2003]. Several such measures, most of which are related to extremal problems, are proposed and investigated.

In [Guo 2012], we found a family of measures of asymmetry as ${ }_{p}(\cdot)$ for convex bodies, called the $p$-measures of asymmetry $(1 \leq p \leq \infty)$ (see definition below), which have the well-known Minkowski measure as a particular case. It turns out that $p$-measures do share some nice properties with the Minkowski measure and might be useful for further research.

As shown in [Guo 2012], for any convex body $C$ and $1 \leq p<\infty$, we have $\operatorname{as}_{p}(C) \leq \operatorname{as}_{\infty}(C)$ in general, and equality holds if $C$ is a symmetric convex body or a simplex. Equality also holds for some nontrivial (i.e., neither symmetric nor a simplex) convex bodies (see examples in Remark 2.3 below). Since, in some sense,

Project supported by the National Natural Science Foundation of China (No. 11271282; 11371013). MSC2010: 52A20, 52A38, 52A39, 52A40.

Keywords: Convex bodies, $p$-measure of asymmetry, Mixed volume, Mixed area measure. 
$\operatorname{as}_{\infty}(C)$ is a maximal value and $\operatorname{as}_{1}(C)$ is a mean of a certain function related to $C$, defined on the unit sphere, it is interesting to consider the following question: Under what conditions is it true that $\operatorname{as}_{1}(C)=\operatorname{as}_{\infty}(C)$ (and therefore that all as ${ }_{p}(C)$ coincide)?

In this article, we reveal more properties of 1-measure and $\infty$-measure and give some calculating formulas of $p$-measures, in particular, for coproducts of convex bodies (see definition below). We will also formulate some questions related to the question above. From now on, we will simply write asymmetry instead of central asymmetry.

\section{Preliminary}

Let $\mathbb{R}^{n}$ denote the usual $n$-dimensional Euclidean space and $\langle\cdot, \cdot\rangle$ the canonical inner product on $\mathbb{R}^{n}$. Denote by $\mathcal{K}^{n}$ the class of all convex bodies (compact convex sets with nonempty interior) in $\mathbb{R}^{n}$, by $\operatorname{Aff}\left(\mathbb{R}^{n}\right)$ the family of all affine maps from $\mathbb{R}^{n}$ to $\mathbb{R}^{n}$, and by aff $\left(\mathbb{R}^{n}\right)$ the family of all affine functionals on $\mathbb{R}^{n}$, which forms an $(n+1)$-dimensional linear space under the ordinary addition and scalar multiplication of functions.

We adopt the following notation and terms from [Schneider 1993].

For $C_{1}, \ldots, C_{n} \in \mathcal{K}^{n}$, denote by $V\left(C_{1}, \ldots, C_{n}\right)$ the mixed volume of $C_{1}, \ldots, C_{n}$ and let $V(C[k])$ be an abbreviated notation for

$$
V(C, \stackrel{(k)}{.}, C,-C, \stackrel{(n-k)}{\cdot-}, C), \quad 0 \leq k \leq n \text {. }
$$

Similarly, denote by $S\left(C_{1}, \ldots, C_{n-1}, \cdot\right)$ the mixed area measure (of $C_{1}, \ldots, C_{n-1}$ ) on $\mathbb{S}^{n-1}$, the $(n-1)$-dimensional unit sphere. It is stated in [Schneider 1993] that $V(C[0])=V([n])=V_{n}(C)$, where $V_{n}(\cdot)$ denotes the $n$-dimensional volume, and $S(C, \stackrel{(n-1)}{\cdot-\cdot}, C, \cdot)=S_{n-1}(C, \cdot)$, the surface area measure of $C$ on $\mathbb{S}^{n-1}$.

For $\alpha \in \mathbb{R}$ and $u \in \mathbb{S}^{n-1}$, set $H_{u, \alpha}=\left\{x \in \mathbb{R}^{n} \mid\langle x, u\rangle=\alpha\right\}$ and notice that $H_{u, \alpha}$ is a hyperplane.

For $C \in \mathcal{K}^{n}$ and $x \in \mathbb{R}^{n}$, we define the support function of $C$ based at $x$ by

$$
h_{x}(C, u):=\sup _{x \in C}\langle y-x, u\rangle, \quad \text { for all } u \in \mathbb{S}^{n-1} .
$$

Denote $F(C, u):=C \cap H_{u, h_{x}(C, u)}$, which is independent of $x$ and called the support set (of $C$ ) in the direction $u$.

It is shown in Theorem 5.1.6 of [Schneider 1993] that, for each $x \in \mathbb{R}^{n}$,

$$
\begin{aligned}
V(C[n-1]) & =\frac{1}{n} \int_{\mathbb{S}^{n-1}} h_{x}(C,-u) d S_{n-1}(C, u), \\
V_{n}(C) & =\frac{1}{n} \int_{\mathbb{S}^{n-1}} h_{x}(C, u) d S_{n-1}(C, u) .
\end{aligned}
$$


Given $C \in \mathcal{K}^{n}$, for $x \in \operatorname{int}(C)$, we write

$$
\mu_{p}(C, x):= \begin{cases}\left(\int_{\mathbb{S}^{n-1}} \alpha_{x}(C, u)^{p} d m_{x}(C, u)\right)^{1 / p} & \text { if } 1 \leq p<\infty, \\ \sup _{u \in \mathbb{S}^{n-1}} \alpha_{x}(C, u) & \text { if } p=\infty,\end{cases}
$$

where $\alpha_{x}(C, u):=h_{x}(C,-u) / h_{x}(C, u)$ and, for measurable $\omega \subset \mathbb{S}^{n-1}$,

$$
m_{x}(C, \omega):=\frac{\int_{\omega} h_{x}(C, u) d S_{n-1}(C, u)}{n V_{n}(C)}=\frac{\int_{\omega} h_{x}(C, u) d S_{n-1}(C, u)}{\int_{\mathbb{S}^{n-1}} h_{x}(C, u) d S_{n-1}(C, u)},
$$

which is a probability measure on $\mathbb{S}^{n-1}$.

Remark 1.1. If $C$ is a polytope with (all) facets $F\left(C, u_{i}\right), i=1,2, \ldots, m$, where $u_{i}$ are outer normal vectors, then the measures $S_{n-1}(C, \cdot)$ and $m_{x}(C, \cdot)$ are linear combinations of $m$ Dirac measures $\delta_{u_{i}}, i=1,2, \ldots, m$, and so the integrals appearing above are just finite sums.

Definition [Guo 2012]. For $C \in \mathcal{K}^{n}$, we define its $p$-measure of asymmetry $(1 \leq p \leq \infty)$ as $p(C)$ by

$$
\operatorname{as}_{p}(C):=\inf _{x \in \operatorname{int}(C)} \mu_{p}(C, x) .
$$

A point $x \in \operatorname{int}(C)$ satisfying $\mu_{p}(C, x)=\operatorname{as}_{p}(C)$ is called a $p$-critical point of $C$. The set of all $p$-critical points is called the $p$-critical set (of $C$ ), denoted by $\mathcal{C}_{p}(C)$.

Remark 1.2. (i) The measure

$$
\operatorname{as}_{\infty}(C)=\inf _{x \in \operatorname{int}(C)} \sup _{u \in \mathbb{S}^{n-1}} \frac{h_{x}(C,-u)}{h_{x}(C, u)}
$$

is nothing else but the Minkowski measure of asymmetry (of $C$ ). The measure as ${ }_{1}(C)$ is the (minimal) mean of $h_{x}(C,-u) / h_{x}(C, u)$ (against $m_{x}(C, \cdot)$ ) among all $x \in \operatorname{int}(C)$, which is in fact independent of $x$ (i.e., $\mu_{1}(C, x)=\operatorname{as}_{1}(C)$ for all $x \in \operatorname{int}(C)$; see $(*))$.

(ii) It is shown in [Guo 2012] that if defining, for any $\varepsilon \geq 0$,

$$
\phi(\varepsilon):=\left(\frac{V_{n}(C-\varepsilon C)}{V_{n}(C)}\right)^{1 / n},
$$

then $\operatorname{as}_{1}(C)=\phi_{+}^{\prime}(0)$ (in fact, this is the definition of 1-measure in [Guo 2012]).

(iii) By definition 1 and (*) above, we see that $\operatorname{as}_{1}(C)=V(C[n-1]) / V_{n}(C)$.

(iv) It is proved in [Guo 2012] that, for $1<p<\infty, \mathcal{C}_{p}$ is a singleton.

One of the main results in [Guo 2012] is the following theorem. 
Theorem 1.3. For any $1 \leq p, q \leq \infty$, the following statements are true:

(i) $\operatorname{as}_{p}(\cdot)$ is affinely invariant, i.e., $\operatorname{as}_{p}(C)=\operatorname{as}_{p}(\mathrm{~T}(C))$ for any $C \in \mathcal{K}^{n}$ and any invertible $\mathrm{T} \in \operatorname{Aff}\left(\mathbb{R}^{n}\right)$.

(ii) $\operatorname{as}_{p}(C) \leq \operatorname{as}_{q}(C)$, for any $C \in \mathcal{K}^{n}$ and $1 \leq p<q \leq \infty$.

(iii) $1 \leq \operatorname{as}_{p}(C) \leq n, \operatorname{as}_{p}(C)=1$ if and only if $C$ is symmetric, and $\operatorname{as}_{p}(C)=n$ if and only if $C$ is a simplex.

\section{The 1-measure of asymmetry for coproducts of convex bodies}

In [Guo 2012] we showed that $p$-measures do share some nice properties with Minkowski's measure. Here we will present more.

We first recall a conclusion in [Guo and Kaijser 2002]: for any $(n-1)$-dimensional convex set $C \subset \mathbb{R}^{n}, \operatorname{as}_{\infty}\left(\widehat{C}_{z}\right)=\operatorname{as}_{\infty}(C)+1$, where $\widehat{C}_{z}:=\operatorname{conv}(C, z)$ is the convex hull of $C \cup\{z\}$ (called the cone with vertex $z$ and base $C$ ) and $z$ is not in the affine hull of $C$ (where as ${ }_{\infty}(C)$ is computed in the $(n-1)$-dimensional space). Furthermore, all $\infty$-critical points $x^{*}$ of $\widehat{C}_{z}$ are of the form

$$
x^{*}=\frac{1}{2+\operatorname{as}_{\infty}(C)} z+\left(\frac{1+\operatorname{as}_{\infty}(C)}{2+\operatorname{as}_{\infty}(C)}\right) x^{\prime},
$$

where $x^{\prime}$ is an $\infty$-critical point of $C$.

We show that a similar conclusion holds for 1-measure but not for 2-measure. Further, we extend the result to the so-called coproducts of subsets which are a generalization of cones (see definition below).

Let us start with cones.

Theorem 2.1. Let $C, z$ be as above. Then

(i) $\operatorname{as}_{1}\left(\widehat{C}_{z}\right)=\operatorname{as}_{1}(C)+1$.

(ii) $\operatorname{as}_{2}\left(\widehat{C}_{z}\right)^{2}=\operatorname{as}_{2}(C)^{2}+2 \sqrt{\operatorname{as}_{2}(C)^{2}+2 \mathrm{as}_{1}(C)+1}-1$. Consequently we have $\operatorname{as}_{2}\left(\widehat{C}_{z}\right) \leq \operatorname{as}_{2}(C)+1$, where equality holds if and only if $\operatorname{as}_{2}(C)=\operatorname{as}_{1}(C)$.

To prove Theorem 2.1, the following lemma is needed.

Lemma 2.2. Let $C, z$ be the same as in Theorem 2.1. For $x$ in $\mathrm{ri}(C)$, the relative interior of $C$, if $z_{\lambda}=\lambda z+(1-\lambda) x(0<\lambda<1)$, then, for any $1 \leq p<\infty$,

$$
\mu_{p}\left(\widehat{C}_{z}, z_{\lambda}\right)^{p}=\lambda\left(\frac{1-\lambda}{\lambda}\right)^{p}+\frac{1}{(1-\lambda)^{p-1}} \int_{\mathbb{S}^{n-2}}\left(\lambda+\alpha_{x}(C, u)\right)^{p} d m_{x}(C, u) .
$$

Proof. Since the family of $(n-1)$-dimensional polytopes is dense in $\mathcal{K}^{n-1}$, and $C_{i} \rightarrow C$ implies $\widehat{C}_{i} \rightarrow \widehat{C}$ (with respect to the Hausdorff metric), and $S_{n-1}(C, \cdot)$ is weakly continuous, we may assume, without loss of generality, that

$$
C=\operatorname{conv}\left(v_{1}, \ldots, v_{l}\right)
$$


is an $(n-1)$-dimensional polytope, where $v_{i}$ are (all) vertices of $C$.

Thus

$$
\widehat{C}=\operatorname{conv}\left(v_{1}, \ldots, v_{l}, z\right)
$$

is the $n$-dimensional polytope with vertices $v_{1}, \ldots, v_{l}$ and $z$. Furthermore, if all facets of $C$ are $F_{i}(1 \leq i \leq m)$, then all facets of $\widehat{C}$ are $\widehat{F}_{i}=\operatorname{conv}\left(F_{i}, z\right)(1 \leq i \leq m)$ and $C$. We denote by $\tilde{u}_{0} \in \mathbb{S}^{n-1}$ the outer normal vector of $C$ (as a facet of $\widehat{C}$ ), by $\tilde{u}_{i} \in \mathbb{S}^{n-1}$ the outer normal vector of $\widehat{F}_{i}$, and by $u_{i} \in \mathbb{S}^{n-2} \equiv H^{*} \cap \mathbb{S}^{n-1}$, where $H^{*}$ denotes the $(n-1)$-dimensional subspace parallel to the affine hull $H$ of $C$, the outer normal vector of $F_{i}$ (as a facet of $C$ ).

Use the fact that $\left\langle z-x, \tilde{u}_{i}\right\rangle=h_{x}\left(\widehat{C}, \tilde{u}_{i}\right)$ to observe that

$$
\begin{aligned}
h_{z \lambda}\left(\widehat{C}, \tilde{u}_{i}\right) & =\sup _{y \in \widehat{C}}\left\langle y-z_{\lambda}, \tilde{u}_{i}\right\rangle=\sup _{y \in \widehat{C}}\left\langle\left(y+x-z_{\lambda}\right)-x, \tilde{u}_{i}\right\rangle \\
& =\sup _{y^{\prime} \in \widehat{C}+x-z_{\lambda}}\left\langle y^{\prime}-x, \tilde{u}_{i}\right\rangle=h_{x}\left(\widehat{C}+x-z_{\lambda}, \tilde{u}_{i}\right) \\
& =h_{x}\left(\widehat{C}+\lambda(x-z), \tilde{u}_{i}\right)=h_{x}\left(\widehat{C}, \tilde{u}_{i}\right)-\lambda\left\langle z-x, \tilde{u}_{i}\right\rangle \\
& =(1-\lambda) h_{x}\left(\widehat{C}, \tilde{u}_{i}\right) .
\end{aligned}
$$

This, together with the fact that $h_{x}\left(\widehat{C}, \tilde{u}_{i}\right)+h_{x}\left(\widehat{C},-\tilde{u}_{i}\right)$ is just the width of $\widehat{C}$ along $\tilde{u}_{i}$ and does not depend on the choice of $x$, in turn leads to

$$
h_{z_{\lambda}}\left(\widehat{C},-\tilde{u}_{i}\right)=\lambda h_{x}\left(\widehat{C}, \tilde{u}_{i}\right)+h_{x}\left(\widehat{C},-\tilde{u}_{i}\right) .
$$

Finally we get

$$
\begin{aligned}
\alpha_{z_{\lambda}}\left(\widehat{C}, \tilde{u}_{i}\right) & =\frac{h_{z_{\lambda}}\left(\widehat{C},-\tilde{u}_{i}\right)}{h_{z_{\lambda}}\left(\widehat{C}, \tilde{u}_{i}\right)}=\frac{\lambda}{1-\lambda}+\frac{1}{1-\lambda} \frac{h_{x}\left(\widehat{C},-\tilde{u}_{i}\right)}{h_{x}\left(\widehat{C}, \tilde{u}_{i}\right)} \\
& =\frac{\lambda}{1-\lambda}+\frac{1}{1-\lambda} \frac{h_{x}\left(C,-u_{i}\right)}{h_{x}\left(C, u_{i}\right)}=\frac{\lambda}{1-\lambda}+\frac{1}{1-\lambda} \alpha_{x}\left(C, u_{i}\right) .
\end{aligned}
$$

Furthermore,

$$
\begin{aligned}
h_{z_{\lambda}}\left(\widehat{C}, \tilde{u}_{i}\right) V_{n-1}\left(\widehat{F}_{i}\right) & =(1-\lambda) h_{x}\left(\widehat{C}, \tilde{u}_{i}\right) V_{n-1}\left(\widehat{F}_{i}\right)=(1-\lambda) n V_{n}\left(\widehat{C}_{i}\right) \\
& =(1-\lambda)\left(h_{z_{\lambda}}\left(\widehat{C}, u_{0}\right)+h_{z_{\lambda}}\left(\widehat{C},-u_{0}\right)\right) V_{n-1}\left(C_{i}\right) \\
& =(1-\lambda)\left(h_{z_{\lambda}}\left(\widehat{C}, u_{0}\right)+h_{z_{\lambda}}\left(\widehat{C},-u_{0}\right)\right) \frac{h_{x}\left(C, u_{i}\right)}{n-1} V_{n-2}\left(F_{i}\right),
\end{aligned}
$$

where $\widehat{C}_{i}$ and $C_{i}$ denote, respectively, the $n$-dimensional body $\operatorname{conv}\left(z, x, F_{i}\right)$ and the $(n-1)$-dimensional body $\operatorname{conv}\left(x, F_{i}\right)$.

Now, by (2-1), (2-2) and the fact that

$$
n V_{n}(\widehat{C})=\left(h_{z_{\lambda}}\left(\widehat{C}, u_{0}\right)+h_{z_{\lambda}}\left(\widehat{C},-u_{0}\right)\right) V_{n-1}(C),
$$


it follows that

$$
\begin{aligned}
\mu_{p}\left(\widehat{C}, z_{\lambda}\right)^{p} \\
=\int_{\mathbb{S}^{n-1}} \alpha_{z_{\lambda}}(\widehat{C}, u)^{p} d m_{z_{\lambda}}(\widehat{C}, u) \\
\quad=\frac{\alpha_{z_{\lambda}}\left(\widehat{C}, u_{0}\right)^{p} h_{z_{\lambda}}\left(\widehat{C}, u_{0}\right) V_{n-1}(C)+\sum_{i=1}^{m} \alpha_{z_{\lambda}}\left(\widehat{C}, \tilde{u}_{i}\right)^{p} h_{z_{\lambda}}\left(\widehat{C}, \tilde{u}_{i}\right) V_{n-1}\left(\widehat{F}_{i}\right)}{n V_{n}(\widehat{C})} \\
=\frac{\alpha_{z_{\lambda}}\left(\widehat{C}, u_{0}\right)^{p} h_{z_{\lambda}}\left(\widehat{C}, u_{0}\right)}{h_{z_{\lambda}}\left(\widehat{C}, u_{0}\right)+h_{z_{\lambda}}\left(\widehat{C},-u_{0}\right)}+\frac{\frac{1-\lambda}{n-1} \sum_{i=1}^{m} \alpha_{z_{\lambda}}\left(\widehat{C}, \tilde{u}_{i}\right)^{p} h_{x}\left(C, u_{i}\right) V_{n-2}\left(F_{i}\right)}{V_{n-1}(C)} \\
=\lambda\left(\frac{1-\lambda}{\lambda}\right)^{p}+\frac{1}{(1-\lambda)^{p-1}} \int_{\mathbb{S}^{n-2}}\left(\lambda+\alpha_{x}(C, u)\right)^{p} d m_{x}(C, u),
\end{aligned}
$$

where we used the equalities $h_{z_{\lambda}}\left(\widehat{C}, u_{0}\right) /\left(h_{z_{\lambda}}\left(\widehat{C}, u_{0}\right)+h_{z_{\lambda}}\left(\widehat{C},-u_{0}\right)\right)=\lambda$ and $\alpha_{z_{\lambda}}\left(\widehat{C}, u_{0}\right)=(1-\lambda) / \lambda$.

Proof of Theorem 2.1. (i) In Lemma 2.2, taking $p=1$, we have, for any $x \in \operatorname{ri}(C)$, $0<\lambda<1$,

$$
\begin{aligned}
\operatorname{as}_{1}\left(\widehat{C}_{z}\right) & =1-\lambda+\int_{\mathbb{S}^{n-2}}\left(\lambda+\alpha_{x}(C, u)\right) d m_{x}(C, u) \\
& =1+\int_{\mathbb{S}^{n-2}} \alpha_{x}(C, u) d m_{x}(C, u)=1+\operatorname{as}_{1}(C) .
\end{aligned}
$$

(ii) In Lemma 2.2, taking $p=2$ and noticing $\mu_{1}(C, x)=\operatorname{as}_{1}(C)$, we have

$$
\begin{aligned}
\mu_{2}\left(\widehat{C}, z_{\lambda}\right)^{2} & =\frac{(1-\lambda)^{2}}{\lambda}+\frac{1}{1-\lambda} \int_{\mathbb{S}^{n-2}}\left(\lambda+\alpha_{x}(C, u)\right)^{2} d m_{x}(C, u) \\
& =\frac{(1-\lambda)^{2}}{\lambda}+\frac{\lambda^{2}}{1-\lambda}+\frac{2 \lambda}{1-\lambda} \operatorname{as}_{1}(C)+\frac{1}{1-\lambda} \mu_{2}(C, x)^{2}=: A(\lambda) .
\end{aligned}
$$

Letting

$$
A^{\prime}(\lambda)=\frac{\left(\mu_{2}(C, x)^{2}+2 \mathrm{as}_{1}(C)\right) \lambda^{2}+2 \lambda-1}{\lambda^{2}(1-\lambda)^{2}}=0,
$$

we get $\lambda_{0}=\left(\sqrt{\mu_{2}(C, x)^{2}+2 \operatorname{as}_{1}(C)+1}+1\right)^{-1}$. Thus, with an elementary computation, it follows that

$$
\begin{aligned}
\min _{0<\lambda<1} \mu_{2}\left(\widehat{C}, z_{\lambda}\right)^{2} & =A\left(\lambda_{0}\right)=\mu_{2}\left(\widehat{C}, z_{\lambda_{0}}\right)^{2} \\
& =\mu_{2}(C, x)^{2}+2 \sqrt{\mu_{2}(C, x)^{2}+2 \operatorname{as}_{1}(C)+1}-1 .
\end{aligned}
$$

Therefore

$$
\operatorname{as}_{2}\left(\widehat{C}_{z}\right)^{2}=\min _{x \in \operatorname{ri}(C)} \min _{0<\lambda<1} \mu_{2}\left(\widehat{C}, z_{\lambda}\right)^{2}=\operatorname{as}_{2}(C)^{2}+2 \sqrt{\operatorname{as}_{2}(C)^{2}+2 \operatorname{as}_{1}(C)+1}-1 .
$$


Since $\operatorname{as}_{1}(C) \leq \operatorname{as}_{2}(C)$ by Theorem 1.3 , we get

$$
\operatorname{as}_{2}\left(\widehat{C}_{z}\right)^{2} \leq \operatorname{as}_{2}(C)^{2}+2 \sqrt{\operatorname{as}_{2}(C)^{2}+2 \operatorname{as}_{2}(C)+1}-1=\left(\operatorname{as}_{2}(C)+1\right)^{2}
$$

which implies that $\operatorname{as}_{2}\left(\widehat{C}_{z}\right) \leq \operatorname{as}_{2}(C)+1$ and that equality holds if and only if $\operatorname{as}_{2}(C)=\operatorname{as}_{1}(C)$.

Remark 2.3. Theorem 2.1 indicates that there are nontrivial $C \in \mathcal{K}^{n}(n \geq 4)$ such that as ${ }_{1}(C)=\operatorname{as}_{\infty}(C)$ : in $\mathbb{R}^{n}$, taking a symmetric $D \in \mathcal{K}^{n-2}$ and forming $\widehat{D}_{y} \in \mathcal{K}^{n-1}$, for each $C:=\operatorname{conv}\left(z, \widehat{D}_{y}\right) \in \mathcal{K}^{n}$, we have, by Theorems 1.3 and 2.1 and Theorem 2 in [Guo and Kaijser 2002], $\operatorname{as}_{1}(C)=\operatorname{as}_{2}(C)=\operatorname{as}_{\infty}(C)=3$, while clearly $C$ is neither a symmetric convex body nor a simplex.

Now we introduce the so-called coproduct of subsets in different spaces and then generalize Theorem 2.1.

Definition. Given $C \subset \mathbb{R}^{m}$ and $D \subset \mathbb{R}^{n}$ ( $\left.m, n \geq 0\right)$, we define the coproduct body $C \amalg D \subset \mathbb{R}^{m+n+1}$ as

$C \amalg D:=\bigcup_{0 \leq \lambda \leq 1}(1-\lambda) C \times \lambda D \times\{\lambda\}=\{((1-\lambda) x, \lambda y, \lambda) \mid x \in C, y \in D, 0 \leq \lambda \leq 1\}$.

Remark 2.4. (i) If both $C$ and $D$ are convex, then $C \amalg D=\operatorname{conv}(C \cup \tilde{D})$, where $\tilde{D}=\{0\} \times D \times\{1\}=\{(0, y, 1) \mid y \in D\}$ (in particular, $C \amalg D$ is convex). For example, $[-1,1] \amalg[-1,1]=\operatorname{conv}\{(1,0,0),(-1,0,0),(0,1,1),(0,-1,1)\}$, a 3-dimensional simplex. In general, $[a, b] \amalg[c, d]$ is a 3-dimensional simplex.

(ii) If $C=\{v\}$ is a singleton and $D$ is convex, then $C \amalg D$ reduces to the cone with vertex $v$ and base $D$.

The next proposition, which may be checked easily, shows that, in a sense, the coproduct operation is the dual of product operation. For $C \in \mathcal{K}^{n}$, denote

$$
C^{A}=\left\{f \in \operatorname{aff}\left(\mathbb{R}^{n}\right) \mid f(C) \subset[-1,1]\right\} .
$$

Proposition 2.5. For any $C \in \mathcal{K}^{m}$ and $D \in \mathcal{K}^{n}$ under the correspondence

$$
(C \amalg D)^{A} \ni f \longleftrightarrow\left(f_{\mid C}, f_{\mid D}\right) \in C^{A} \times D^{A},
$$

where $\left(f_{\mid C}, f_{\mid D}\right)(((1-\lambda) x, \lambda y, \lambda)):=(1-\lambda) f_{\mid C}(x)+\lambda f_{\mid D}(y)$, we have

$$
(C \amalg D)^{A}=C^{A} \times D^{A} .
$$

Now we can generalize (i) in Theorem 2.1 to the coproduct bodies.

Theorem 2.6. For any $C \in \mathcal{K}^{m}$ and $D \in \mathcal{K}^{n}(m, n \geq 0)$,

$$
\operatorname{as}_{1}(C \amalg D)=\operatorname{as}_{1}(C)+\operatorname{as}_{1}(D)+1,
$$

where we take the convention that $\operatorname{as}_{1}(C)\left(\right.$ or $\left.\operatorname{as}_{1}(D)\right)=0$ if $C($ or $D) \in \mathcal{K}^{0}$. 
In order to prove Theorem 2.6, more lemmas are needed. For any $\lambda \in \mathbb{R}, \varepsilon \geq 0$ and $A \subset \mathbb{R}^{m+n+1}$, denote

$$
\mathbb{H}_{\lambda}:=\mathbb{R}^{m} \times \mathbb{R}^{n} \times\{\lambda\}, \quad A_{\lambda}:=A \cap \mathbb{H}_{\lambda}, \quad A_{\lambda, \varepsilon}:=[A-\varepsilon A]_{\lambda} .
$$

Lemma 2.7. For any $C \in \mathcal{K}^{m}$ and $D \in \mathcal{K}^{n}(m, n \geq 1)$,

$$
V_{m+n+1}(C \amalg D)=B(m+1, n+1) V_{m}(C) V_{n}(D),
$$

where $B(\cdot, \cdot)$ is the Beta function.

Proof. Since $[C \amalg D]_{\lambda}=(1-\lambda) C \times \lambda D \times\{\lambda\}$ for $0 \leq \lambda \leq 1$, we have

$$
V_{m+n}\left([C \amalg D]_{\lambda}\right)=V_{m+n}((1-\lambda) C \times \lambda D)=(1-\lambda)^{m} \lambda^{n} V_{m}(C) V_{n}(D) .
$$

Hence

$$
V_{m+n+1}(C \sqcup D)=\int_{0}^{1}(1-\lambda)^{m} \lambda^{n} V_{m}(C) V_{n}(D) d \lambda=B(m+1, n+1) V_{m}(C) V_{n}(D) .
$$

Lemma 2.8. If $o \in C$ and $o \in D$, then for any $0 \leq \varepsilon<1$ and $0 \leq \lambda \leq 1-\varepsilon$,

$$
\left.V_{m+n}\left([C \amalg D]_{\lambda, \varepsilon}\right)=V_{m+n}(((1-\lambda) C-\varepsilon C)) \times((\lambda+\varepsilon) D-\varepsilon D)\right)-\varepsilon^{2} P^{*}(\lambda, \varepsilon),
$$

where $P^{*}(\lambda, \varepsilon)$ is a polynomial of $\lambda$ and $\varepsilon$.

Proof. Since

$C \amalg D-\varepsilon(C \amalg D)$

$$
\begin{aligned}
& =\bigcup_{0 \leq \mu, \nu \leq 1}((1-\mu) C \times \mu D \times\{\mu\}-\varepsilon(1-v) C \times \varepsilon v D \times\{\varepsilon v\}) \\
& =\bigcup_{0 \leq \mu, \nu \leq 1}((1-\mu) C-\varepsilon(1-v) C) \times(\mu D-\varepsilon v D) \times\{\mu-\varepsilon v\},
\end{aligned}
$$

we have

$$
[C \amalg D]_{\lambda, \varepsilon}=\bigcup_{\mu-\varepsilon \nu=\lambda}((1-\mu) C-\varepsilon(1-v) C) \times(\mu D-\varepsilon \nu D) \times\{\lambda\} .
$$

Thus,

$$
[C \amalg D]_{\lambda, \varepsilon} \supset((1-(\lambda+\varepsilon)) C) \times((\lambda+\varepsilon) D-\varepsilon D) \times\{\lambda\}=: E_{1}
$$

(the set when $v=1$ and so $\mu=\lambda+\varepsilon$ ) and

$$
[C \amalg D]_{\lambda, \varepsilon} \supset((1-\lambda) C-\varepsilon C) \times(\lambda D) \times\{\lambda\}=: E_{2}
$$

(the set when $v=0$ and so $\mu=\lambda$ ). We also have

$$
[C \amalg D]_{\lambda, \varepsilon} \subset((1-\lambda) C-\varepsilon C) \times((\lambda+\varepsilon) D-\varepsilon D) \times\{\lambda\}=: E_{3},
$$


since, for $0 \leq \mu, v \leq 1$ with $\mu-\varepsilon v=\lambda$ (notice that $\lambda \leq \mu \leq \lambda+\varepsilon$ and that $o \in C$, $o \in D)$,

$$
\begin{gathered}
(1-\mu) C-\varepsilon(1-v) C \subset(1-\lambda) C-\varepsilon C, \\
\mu D-\varepsilon v D \subset \mu D-\varepsilon D \subset(\lambda+\varepsilon) D-\varepsilon D .
\end{gathered}
$$

Now, setting

$$
P(\lambda, \varepsilon):=V_{m+n}\left(E_{3}\right)-V_{m+n}\left([C \amalg D]_{\lambda, \varepsilon}\right),
$$

which is a polynomial of $\lambda$ and $\varepsilon$, we have by (2-3), (2-4), (2-5) and the fact that $(1-\lambda-\varepsilon) C \subset(1-\lambda) C-\varepsilon C, \lambda D \subset(\lambda+\varepsilon) D-\varepsilon D$,

$$
\begin{aligned}
0 & \leq P(\lambda, \varepsilon) \\
& \leq V_{m+n}\left(E_{3}\right)-V_{m+n}\left(E_{1} \cup E_{2}\right) \\
& =V_{m+n}\left(E_{3}\right)-V_{m+n}\left(E_{1}\right)-V_{m+n}\left(E_{2}\right)+V_{m+n}\left(E_{1} \cap E_{2}\right) .
\end{aligned}
$$

By the polynomial expansion of the Minkowski sum (see Theorem 5.1.6 in [Schneider 1993]),

$$
\begin{aligned}
V_{m+n}\left(E_{3}\right)= & V_{m}((1-\lambda) C-\varepsilon C) V_{n}((\lambda+\varepsilon) D-\varepsilon D) \\
= & \left((1-\lambda)^{m} V_{m}(C)+m \varepsilon(1-\lambda)^{m-1} V(C[m-1])+\varepsilon^{2} P_{1}^{\prime}(\lambda, \varepsilon)\right) \\
& \times\left((\lambda+\varepsilon)^{n} V_{n}(D)+n \varepsilon(\lambda+\varepsilon)^{n-1} V(D[n-1])+\varepsilon^{2} P_{1}^{\prime \prime}(\lambda, \varepsilon)\right) \\
= & (1-\lambda)^{m}(\lambda+\varepsilon)^{n} V_{m}(C) V_{n}(D) \\
& +n \varepsilon(1-\lambda)^{m}(\lambda+\varepsilon)^{n-1} V_{m}(C) V(D[n-1]) \\
& +m \varepsilon(1-\lambda)^{m-1}(\lambda+\varepsilon)^{n} V(C[m-1]) V_{n}(D)+\varepsilon^{2} P_{1}(\lambda, \varepsilon),
\end{aligned}
$$

$$
\begin{aligned}
V_{m+n}\left(E_{1}\right)= & V_{m}((1-\lambda-\varepsilon) C) V_{n}((\lambda+\varepsilon) D-\varepsilon D) \\
= & (1-\lambda-\varepsilon)^{m} V_{m}(C) \\
& \quad \times\left((\lambda+\varepsilon)^{n} V_{n}(D)+n \varepsilon(\lambda+\varepsilon)^{n-1} V(D[n-1])+\varepsilon^{2} P_{2}^{\prime}(\lambda, \varepsilon)\right) \\
= & (1-\lambda-\varepsilon)^{m}(\lambda+\varepsilon)^{n} V_{m}(C) V_{n}(D) \\
& \quad+n \varepsilon(1-\lambda-\varepsilon)^{m}(\lambda+\varepsilon)^{n-1} V_{m}(C) V(D[n-1])+\varepsilon^{2} P_{2}(\lambda, \varepsilon),
\end{aligned}
$$

$$
\begin{aligned}
V_{m+n}\left(E_{2}\right)= & V_{m}((1-\lambda) C-\varepsilon C) V_{n}(\lambda D) \\
= & \left((1-\lambda)^{m} V_{m}(C)+m \varepsilon(1-\lambda)^{m-1} V(C[m-1])+\varepsilon^{2} P_{3}^{\prime}(\lambda, \varepsilon)\right) \lambda^{n} V_{n}(D) \\
= & (1-\lambda)^{m} \lambda^{n} V_{m}(C) V_{n}(D) \\
& \quad+m \varepsilon(1-\lambda)^{m-1} \lambda^{n} V(C[m-1]) V_{n}(D)+\varepsilon^{2} P_{3}(\lambda, \varepsilon),
\end{aligned}
$$$$
V_{m+n}\left(E_{1} \cap E_{2}\right)=V_{m}((1-\lambda-\varepsilon) C) V_{n}(\lambda D)=(1-\lambda-\varepsilon)^{m} \lambda^{n} V_{m}(C) V_{n}(D),
$$ 
where $P_{i}^{\prime}, P_{i}^{\prime \prime}, P_{i}$ are polynomials of $\lambda$ and $\varepsilon$, and

$$
\begin{aligned}
(1-\lambda)^{m}(\lambda & +\varepsilon)^{n}-(1-\lambda-\varepsilon)^{m}(\lambda+\varepsilon)^{n}-(1-\lambda)^{m} \lambda^{n}+(1-\lambda-\varepsilon)^{m} \lambda^{n} \\
& =\left((1-\lambda)^{m}-(1-\lambda-\varepsilon)^{m}\right)(\lambda+\varepsilon)^{n}-\left((1-\lambda)^{m}-(1-\lambda-\varepsilon)^{m}\right) \lambda^{n} \\
& =\left((1-\lambda)^{m}-(1-\lambda-\varepsilon)^{m}\right)\left((\lambda+\varepsilon)^{n}-\lambda^{n}\right) \\
& =\varepsilon^{2} Q_{1}(\lambda, \varepsilon),
\end{aligned}
$$

$$
\begin{aligned}
n \varepsilon(1-\lambda)^{m}(\lambda+\varepsilon)^{n-1}-n \varepsilon(1-\lambda-\varepsilon)^{m}(\lambda+\varepsilon)^{n-1} & \\
= & n \varepsilon\left((1-\lambda)^{m}-(1-\lambda-\varepsilon)^{m}\right)(\lambda+\varepsilon)^{n-1} \\
= & \varepsilon^{2} Q_{2}(\lambda, \varepsilon), \\
& \quad m \varepsilon(1-\lambda)^{m-1}\left((\lambda+\varepsilon)^{n}-\lambda^{n}\right)=\varepsilon^{2} Q_{3}(\lambda, \varepsilon),
\end{aligned}
$$

where $Q_{i}$ are polynomials of $\lambda$ and $\varepsilon$. Thus

$$
V_{m+n}\left(E_{3}\right)-V_{m+n}\left(E_{1}\right)-V_{m+n}\left(E_{2}\right)+V_{m+n}\left(E_{1} \cap E_{2}\right)=\varepsilon^{2} Q(\lambda, \varepsilon)
$$

for some polynomial $Q(\lambda, \varepsilon)$, and in turn $P(\lambda, \varepsilon)=\varepsilon^{2} P^{*}(\lambda, \varepsilon)$ for some polynomial $P^{*}(\lambda, \varepsilon)$.

Lemma 2.9. For any $C \in \mathcal{K}^{m}$ and $D \in \mathcal{K}^{n}$ with $o \in C, o \in D(m, n \geq 1)$,

$$
\begin{aligned}
\frac{d}{d \varepsilon}\left(\int_{0}^{1-\varepsilon} V_{m+n}\left([C \amalg D]_{\lambda, \varepsilon}\right) d \lambda\right)_{\mid \varepsilon=0} \\
=(m+n+1) B(m+1, n+1) \\
\quad \times\left(V_{m}(C) V_{n}(D)+V(C[m-1]) V_{n}(D)+V_{m}(C) V(D[n-1])\right) .
\end{aligned}
$$

Proof. By Lemma 2.8 and (2-7), we have

$$
\begin{aligned}
V_{m+n} & \left([C \amalg D]_{\lambda, \varepsilon}\right) \\
= & V_{m+n}\left(E_{3}\right)-\varepsilon^{2} P^{*}(\lambda, \varepsilon) \\
= & (1-\lambda)^{m}(\lambda+\varepsilon)^{n} V_{m}(C) V_{n}(D)+m \varepsilon(1-\lambda)^{m-1}(\lambda+\varepsilon)^{n} V(C[m-1]) V_{n}(D) \\
& \quad+n \varepsilon(\lambda+\varepsilon)^{n-1}(1-\lambda)^{m} V_{m}(C) V(D[n-1])+\varepsilon^{2} P_{1}(\lambda, \varepsilon)-\varepsilon^{2} P^{*}(\lambda, \varepsilon) .
\end{aligned}
$$

Thus, since

$$
\begin{gathered}
\frac{d}{d \varepsilon}\left(\int_{0}^{1-\varepsilon}(1-\lambda)^{m}(\lambda+\varepsilon)^{n} d \lambda\right)_{\mid \varepsilon=0}=n B(m+1, n), \\
\frac{d}{d \varepsilon}\left(\varepsilon \int_{0}^{1-\varepsilon}(1-\lambda)^{m-1}(\lambda+\varepsilon)^{n} d \lambda\right)_{\mid \varepsilon=0}=B(m, n+1),
\end{gathered}
$$




$$
\begin{aligned}
\frac{d}{d \varepsilon}\left(\varepsilon \int_{0}^{1-\varepsilon}(1-\lambda)^{m}(\lambda+\varepsilon)^{n-1} d \lambda\right)_{\mid \varepsilon=0} & =B(m+1, n), \\
\frac{d}{d \varepsilon}\left(\varepsilon^{2} \int_{0}^{1-\varepsilon}\left(P_{1}(\lambda, \varepsilon)-P^{*}(\lambda, \varepsilon)\right) d \lambda\right)_{\mid \varepsilon=0} & =0,
\end{aligned}
$$

and because $m B(m, n+1)=n B(m+1, n)=(m+n+1) B(m+1, n+1)$, we get

$$
\begin{aligned}
\frac{d}{d \varepsilon}\left(\int_{0}^{1-\varepsilon}\right. & \left.V_{m+n}\left([C \amalg D]_{\lambda, \varepsilon}\right) d \lambda\right)_{\mid \varepsilon=0} \\
= & n B(m+1, n) V_{m}(C) V_{n}(D)+m B(m, n+1) V(C[m-1]) V_{n}(D) \\
= & (m+n+1) B(m+1, n+1) \\
& \quad \times\left(V_{m}(C) V_{n}(D)+V(C[m-1]) V_{n}(D)+V_{m}(C) V(D[n-1])\right) .
\end{aligned}
$$

The following simple fact will be needed in the proof of Theorem 2.6.

Fact 2.10. Suppose $0 \leq u(t) \leq v(t)$ and $u(0)=v(0)=0$. If

$$
{\frac{d v(t)}{d t^{+}}}_{\mid t=0}=0
$$

then

$$
0 \leq \lim _{t \rightarrow 0^{+}} \frac{u(t)-u(0)}{t-0} \leq \lim _{t \rightarrow 0^{+}} \frac{v(t)-v(0)}{t-0}=0,
$$

i.e.,

$$
{\frac{d u(t)}{d t^{+}}}_{\mid t=0}=0 \quad\left(\text { or } \frac{d u(t)}{d t}_{\mid t=0}=0 \text { if it exists }\right),
$$

where $d / d t^{+}$denotes the right derivative.

Proof of Theorem 2.6. If $m=n=0$, then $\operatorname{as}_{1}(C)=\operatorname{as}_{1}(D)=0$ and $C \amalg D$ is just the segment with ends $o$ and $(0,0,1)$. Hence $\operatorname{as}_{1}(C \amalg D)=1=\operatorname{as}_{1}(C)+\operatorname{as}_{1}(D)+1$. If $m=0, n \geq 1$ (or $m \geq 1, n=0$ ), it reduces to (i) in Theorem 2.1 (see (ii) in Remark 2.4).

Now we assume $m, n \geq 1$ and $o \in C, o \in D$ ( since $\operatorname{as}_{1}(\cdot)$ is affine invariant). Notice that $C \amalg D-\varepsilon(C \amalg D)$ is located in between $\mathbb{H}_{-\varepsilon}$ and $\dddot{H}_{1}$ since $C \amalg D$ is located in between $\mathbb{H}_{0}$ and $\mathfrak{H}_{1}$.

By the polynomial expansion of the Minkowski sum, we have that

$$
\begin{aligned}
(m+n+1) & V((C \amalg D)[m+n]) \\
= & \frac{d}{d \varepsilon} V_{m+n+1}(C \amalg D-\varepsilon C \amalg D)_{\mid \varepsilon=0} \\
= & \frac{d}{d \varepsilon}\left[\left(\int_{-\varepsilon}^{0}+\int_{0}^{1-\varepsilon}+\int_{1-\varepsilon}^{1}\right) V_{m+n}\left([C \amalg D]_{\lambda, \varepsilon}\right) d \lambda\right]_{\mid \varepsilon=0} .
\end{aligned}
$$


In order to compute

$$
\frac{d}{d \varepsilon}\left(\int_{-\varepsilon}^{0} V_{m+n}\left([C \amalg D]_{\lambda, \varepsilon}\right) d \lambda\right)_{\mid \varepsilon=0},
$$

we observe that if $-\varepsilon \leq \lambda \leq 0$ and $\mu-\varepsilon v=\lambda$, then

$$
((1-\mu) C-\varepsilon(1-v) C) \times(\mu D-\varepsilon v D) \subset(C-\varepsilon C) \times((\lambda+\varepsilon)(D-D)+\lambda D),
$$

since $(1-\mu) C-\varepsilon(1-v) C \subset C-\varepsilon C$ and

$$
\mu D-\varepsilon \nu D=\mu D-(\mu-\lambda) D=\mu(D-D)-\lambda D \subset(\lambda+\varepsilon)(D-D)+\lambda D
$$

(notice that $-\lambda \geq 0, o \in D-D$ and that $\mu-\varepsilon v=\lambda$ implies $\mu \leq \lambda+\varepsilon$ ). So (2-9)

$$
0 \leq \int_{-\varepsilon}^{0} V_{m+n}\left([C \amalg D]_{\lambda, \varepsilon}\right) d \lambda \leq \int_{-\varepsilon}^{0} V_{m}(C-\varepsilon C) V_{n}((\lambda+\varepsilon)(D-D)+\lambda D) d \lambda .
$$

Denote $f(\lambda, \varepsilon):=V_{m}(C-\varepsilon C) V_{n}((\lambda+\varepsilon)(D-D)+\lambda D)$, which is a polynomial of $\lambda$ and $\varepsilon$ by the polynomial expansion of the Minkowski sum, and $f(0,0)=0$. Thus

$$
\frac{d}{d \varepsilon}\left(\int_{-\varepsilon}^{0} f(\lambda, \varepsilon) d \lambda\right)_{\mid \varepsilon=0}=f(0,0)=0
$$

which, together with (2-9) and Fact 2.10, leads to

$$
\frac{d}{d \varepsilon}\left(\int_{-\varepsilon}^{0} V_{m+n}\left([C \amalg D]_{\lambda, \varepsilon}\right) d \lambda\right)_{\mid \varepsilon=0}=0 .
$$

Similarly, we have

$$
\frac{d}{d \varepsilon}\left(\int_{1-\varepsilon}^{1} V_{m+n}\left([C \amalg D]_{\lambda, \varepsilon}\right) d \lambda\right)_{\mid \varepsilon=0}=0 .
$$

Now, (2-8), (2-10), (2-11) and Lemma 2.9 show that

$$
\begin{aligned}
(m+n+1) & V((C \amalg D)[m+n]) \\
= & \frac{d}{d \varepsilon}\left(\int_{0}^{1-\varepsilon} V_{m+n}\left([C \amalg D]_{\lambda, \varepsilon}\right) d \lambda\right)_{\mid \varepsilon=0} \\
= & (m+n+1) B(m+1, n+1) \\
& \quad \times\left(V_{m}(C) V_{n}(D)+V(C[m-1]) V_{n}(D)+V_{m}(C) V(D[n-1])\right),
\end{aligned}
$$


which, together with (iii) in Remark 1.2 and Lemma 2.7, leads to

$$
\begin{aligned}
\operatorname{as}_{1}(C \amalg D) & =\frac{V((C \amalg D)[m+n])}{V_{m+n+1}(C \amalg D)} \\
& =\frac{V_{m}(C) V_{n}(D)+V(C[m-1]) V_{n}(D)+V_{m}(C) V(D[n-1])}{V_{m}(C) V_{n}(D)} \\
& =1+\operatorname{as}_{1}(C)+\operatorname{as}_{1}(D) .
\end{aligned}
$$

\section{The Minkowski measure of coproducts of convex bodies}

In this section, we will show that Theorem 2.6 also holds for the well-known Minkowski measure as ${ }_{\infty}$.

First, given $C \in \mathcal{K}^{n}$, for any fixed $x \in \operatorname{int}(C)$, define

$$
\gamma(C, x):=\sup \left\{f(x) \mid f \in C^{a}\right\},
$$

where $C^{a}:=\left\{f \in \operatorname{aff}\left(R^{n}\right) \mid f(C)=[-1,1]\right\}$. It is easy to check (see [Guo 2005]) that $\mu_{\infty}(C, x)=(1+\gamma(C, x)) /(1-\gamma(C, x))$. Defining a measure of asymmetry $\operatorname{As}(C)$ of $C$ by

$$
\operatorname{As}(C)=\inf _{x \in \operatorname{int}(C)} \gamma(C, x),
$$

we have $0 \leq \operatorname{As}(C) \leq(n-1) /(n+1)$ for $C \in \mathcal{K}^{n}$ and

$$
\operatorname{As}(C)=\frac{\operatorname{as}_{\infty}(C)-1}{\operatorname{as}_{\infty}(C)+1} \quad \text { or } \quad \operatorname{as}_{\infty}(C)=\frac{1+\operatorname{As}(C)}{1-\operatorname{As}(C)} .
$$

Then $x$ is an $\infty$-critical point if and only if it is an As-critical point, and it is reasonable to study the Minkowski measure as ${ }_{\infty}(C)$ in terms of $\gamma(C, x)$ and $\operatorname{As}(C)$. Definition. For $C \in \mathcal{K}^{m}$ and $D \in \mathcal{K}^{n}$, we define the affine direct sum of $C^{a}$ and $D^{a}$, $C^{a} \boxplus D^{a} \subset \operatorname{aff}\left(\mathbb{R}^{m}\right) \times \operatorname{aff}\left(\mathbb{R}^{n}\right)$, by

$$
C^{a} \boxplus D^{a}:=\left(\left\{1_{C}\right\} \times D^{a}\right) \cup\left(C^{a} \times\left\{1_{D}\right\}\right),
$$

where $1_{C}$ and $1_{D}$ denote the constant function 1 respectively on $\mathbb{R}^{m}$ and $\mathbb{R}^{n}$.

Under the same correspondence as in Proposition 2.5, $C^{a} \boxplus D^{a}$ can be identified with a subset of $(C \amalg D)^{a}$, and it is easy to check that

$$
C^{a} \boxplus D^{a} \subsetneq(C \amalg D)^{a} \subsetneq(C \amalg D)^{A} .
$$

Lemma 3.1. Given $C \in \mathcal{K}^{m}$ and $D \in \mathcal{K}^{n}$, for any fixed $z=((1-\lambda) x, \lambda y, \lambda)$ in $\operatorname{int}(C \sqcup D)$ (i.e., $x \in \operatorname{ri}(C), y \in \operatorname{ri}(D)$ and $0<\lambda<1$ ),

$$
\gamma_{z}:=\gamma(C \amalg D, z)=\sup _{(f, g) \in(C \sqcup D)^{a}}(f, g)(z)=\sup _{(f, g) \in C^{a} \boxplus D^{a}}(f, g)(z),
$$

where $f \in \operatorname{aff}\left(\mathbb{R}^{m}\right), g \in \operatorname{aff}\left(\mathbb{R}^{n}\right)$ and $(f, g)(z):=(1-\lambda) f(x)+\lambda g(y)$. 
Proof. By a standard compactness argument, there is $\left(f_{0}, g_{0}\right) \in(C \amalg D)^{a}$ such that

$$
\gamma_{z}=\left(f_{0}, g_{0}\right)(z)=(1-\lambda) f_{0}(x)+\lambda g_{0}(y) .
$$

Now we will show that $f_{0}=1_{C}$ and $g_{0} \in D^{a}$ or $g_{0}=1_{C}$ and $f_{0} \in C^{a}$.

To see this, we observe first that $f_{0}(C) \subset[-1,1], g_{0}(D) \subset[-1,1]$ and

$$
\left(f_{0}, g_{0}\right)(C \amalg D)=\operatorname{conv}\left(f_{0}(C) \cup g_{0}(D)\right),
$$

which can be easily checked by the definition of $C \amalg D$ (in fact, this holds for any $\left.(f, g) \in(C \amalg D)^{a}\right)$.

Then we claim that $1 \in f_{0}(C)$ and $1 \in g_{0}(D)$. Suppose it is not true that, say, $1 \notin f_{0}(C)$. Then (3-2) implies that $1 \in g_{0}(D)$ since $\left(f_{0}, g_{0}\right)(C \amalg D)=[-1,1]$, and either -1 is in $f_{0}(C)$ or $g_{0}(D)$. However, we will see that in either case there is a contradiction.

If $-1 \in g_{0}(D)$, then $g_{0} \in D^{a}$. Thus $\left(1_{C}, g_{0}\right) \in(C \amalg D)^{a}$ and we have the inequality $\left(1_{C}, g_{0}\right)(z)>\left(f_{0}, g_{0}\right)(z)$, which contradicts (3-1).

If $-1 \in f_{0}(C)$, then we can find $f_{1} \in C^{a}$ such that $\left\{f_{1}=-1\right\}=\left\{f_{0}=-1\right\}$, which implies that $f_{1}(x)>f_{0}(x)$ (since $1 \notin f_{0}(C)$ ). Thus $\left(f_{1}, g_{0}\right) \in(C \amalg D)^{a}$ and $\left(f_{1}, g_{0}\right)(z)>\left(f_{0}, g_{0}\right)(z)$ which contradicts (3-1) too. Hence we have confirmed our claim.

Now, with a similar argument, we can show that -1 is in $f_{0}(C)$ or $g_{0}(D)$.

If $-1 \in g_{0}(D)$, then $g_{0} \in D^{a}$, and we must have $f_{0}=1_{C}$ since $\left(1_{C}, g_{0}\right) \in(C \amalg D)^{a}$ and $\left(1_{C}, g_{0}\right)(z)>\left(f, g_{0}\right)(z)$ for all $f \neq 1_{C}$. Thus $\left(f_{0}, g_{0}\right)=\left(1_{C}, g_{0}\right) \in C^{a} \boxplus D^{a}$.

Similarly, if $-1 \in f_{0}(C)$, then $g_{0}=1_{D}$ and so $\left(f_{0}, g_{0}\right)=\left(f_{0}, 1_{D}\right) \in C^{a} \boxplus D^{a}$.

Now we can prove the following generalization of Theorem 2 in [Guo and Kaijser 2002].

Theorem 3.2. For any $C \in \mathcal{K}^{m}$ and $D \in \mathcal{K}^{n}(m, n \geq 0)$,

$$
\operatorname{as}_{\infty}(C \amalg D)=\operatorname{as}_{\infty}(C)+\operatorname{as}_{\infty}(D)+1,
$$

where we take the convention that $\operatorname{as}_{\infty}(C)=0$ for $C \in \mathcal{K}^{0}$. Moreover, all $\infty$-critical points $z^{*}$ of $C \amalg D$ have the form

$$
z^{*}=\frac{1-\gamma_{y}}{2-\gamma_{x}-\gamma_{y}} x^{*}+\frac{1-\gamma_{x}}{2-\gamma_{x}-\gamma_{y}} y^{*}
$$

where $x^{*}=(x, 0,0)$ with $x$ being an $\infty$-critical point of $C$, and $y^{*}=(0, y, 1)$ with $y$ being an $\infty$-critical point of $D$, and $\gamma_{x}:=\gamma(C, x), \gamma_{y}:=\gamma(D, y)$.

Proof. If $m=n=0$, the same argument as in the proof of Theorem 2.6 can be applied.

If $m=0, n \geq 1$ or $m \geq 1, n=0$, it reduces to Theorem 2 in [Guo and Kaijser 2002]. 
Now assume $m \geq 1, n \geq 1$. We first prove a general result: for any $\bar{x}:=(x, 0,0)$ with $x \in \operatorname{ri}(C)$ and $\bar{y}:=(0, y, 1)$ with $y \in \operatorname{ri}(D)$,

$$
\min _{z \in(\bar{x}, \bar{y})} \gamma(C \amalg D, z)=\gamma\left(C \amalg D, z_{0}\right)=\frac{1-\gamma_{x} \gamma_{y}}{2-\gamma_{x}-\gamma_{y}},
$$

where $(\bar{x}, \bar{y})$ is the open interval with $\bar{x}, \bar{y}$ as ends and

$$
z_{0}=\frac{1-\gamma_{y}}{2-\gamma_{x}-\gamma_{y}} \bar{x}+\frac{1-\gamma_{x}}{2-\gamma_{x}-\gamma_{y}} \bar{y} .
$$

In fact, for any $(1, g),(f, 1) \in C^{a} \boxplus D^{a}$,

$$
\begin{aligned}
(1, g)\left(z_{0}\right) & =\frac{1-\gamma_{y}}{2-\gamma_{x}-\gamma_{y}}+\frac{1-\gamma_{x}}{2-\gamma_{x}-\gamma_{y}} g(y) \\
& \leq \frac{1-\gamma_{y}}{2-\gamma_{x}-\gamma_{y}}+\frac{1-\gamma_{x}}{2-\gamma_{x}-\gamma_{y}} \gamma_{y}=\frac{1-\gamma_{x} \gamma_{y}}{2-\gamma_{x}-\gamma_{y}}, \\
(f, 1)\left(z_{0}\right) & =\frac{1-\gamma_{y}}{2-\gamma_{x}-\gamma_{y}} f(x)+\frac{1-\gamma_{x}}{2-\gamma_{x}-\gamma_{y}} \\
& \leq \frac{1-\gamma_{y}}{2-\gamma_{x}-\gamma_{y}} \gamma_{x}+\frac{1-\gamma_{x}}{2-\gamma_{x}-\gamma_{y}}=\frac{1-\gamma_{x} \gamma_{y}}{2-\gamma_{x}-\gamma_{y}},
\end{aligned}
$$

with equality in the first formula if $g \in D^{a}$ such that $g(y)=\gamma_{y}$ and equality in the second formula if $f \in C^{a}$ such that $f(x)=\gamma_{x}$. So by Lemma 3.1, we have $\gamma_{z_{0}}=\left(1-\gamma_{x} \gamma_{y}\right) /\left(2-\gamma_{x}-\gamma_{y}\right)$.

Now, for $z=\lambda \bar{x}+(1-\lambda) \bar{y} \in[\bar{x}, \bar{y}]$, if $\lambda>\left(1-\gamma_{y}\right) /\left(2-\gamma_{x}-\gamma_{y}\right)$, we choose $g_{0} \in D^{a}$ such that $g_{0}(y)=\gamma_{y}$. Then

$$
\begin{aligned}
\left(1, g_{0}\right)(z) & =\lambda+(1-\lambda) \gamma_{y}=\left(1-\gamma_{y}\right) \lambda+\gamma_{y} \\
& >\left(1-\gamma_{y}\right) \frac{1-\gamma_{y}}{2-\gamma_{x}-\gamma_{y}}+\gamma_{y}=\frac{1-\gamma_{x} \gamma_{y}}{2-\gamma_{x}-\gamma_{y}}=\gamma_{z_{0}},
\end{aligned}
$$

which implies that $\gamma_{z}>\gamma_{z_{0}}$.

If $\lambda<\left(1-\gamma_{y}\right) /\left(2-\gamma_{x}-\gamma_{y}\right)$, then, noticing that $\gamma_{x}-1<0$, we have

$$
\begin{aligned}
\left(f_{0}, 1\right)(z) & =\lambda \gamma_{x}+(1-\lambda)=\left(\gamma_{x}-1\right) \lambda+1 \\
& >\left(\gamma_{x}-1\right) \frac{1-\gamma_{y}}{2-\gamma_{x}-\gamma_{y}}+1=\frac{1-\gamma_{x} \gamma_{y}}{2-\gamma_{x}-\gamma_{y}}=\gamma_{z_{0}},
\end{aligned}
$$

which also implies that $\gamma_{z}>\gamma_{z_{0}}$. Hence (3-3) is confirmed. 
Finally, since it is easy to check that $\mu_{\infty}(C, x)=(1+\gamma(C, x)) /(1-\gamma(C, x))$, we can use the fact that the function $(1+t) /(1-t)$ is increasing on $[0,1)$, to get

$$
\begin{aligned}
\mu_{\infty}\left(C \amalg D, z_{0}\right) & =\min _{z \in(\bar{x}, \bar{y})} \mu_{\infty}(C \amalg D, z)=\frac{1+\gamma_{z_{0}}}{1-\gamma_{z_{0}}} \\
& =\left(1+\frac{1-\gamma_{x} \gamma_{y}}{2-\gamma_{x}-\gamma_{y}}\right)\left(1-\frac{1-\gamma_{x} \gamma_{y}}{2-\gamma_{x}-\gamma_{y}}\right)^{-1} \\
& =\frac{3-\gamma_{x}-\gamma_{y}-\gamma_{x} \gamma_{y}}{1-\gamma_{x}-\gamma_{y}+\gamma_{x} \gamma_{y}}=\frac{1+\gamma_{x}}{1-\gamma_{x}}+\frac{1+\gamma_{y}}{1-\gamma_{y}}+1 \\
& =\mu_{\infty}(C, x)+\mu_{\infty}(D, y)+1 .
\end{aligned}
$$

It follows that

$\operatorname{as}_{\infty}(C \amalg D)=\min _{x \in \operatorname{ri}(C), y \in \operatorname{ri}(D)}\left(\mu_{\infty}(C, x)+\mu_{\infty}(D, y)+1\right)=\operatorname{as}_{\infty}(C)+\operatorname{as}_{\infty}(D)+1$ and all $\infty$-critical points $z^{*}$ of $C \amalg D$ have the form

$$
z^{*}=\frac{1-\gamma_{y}}{2-\gamma_{x}-\gamma_{y}} x^{*}+\frac{1-\gamma_{x}}{2-\gamma_{x}-\gamma_{y}} y^{*}
$$

where $x^{*}=(x, 0,0)$ with $x$ being an $\infty$-critical point of $C$ and $y^{*}=(0, y, 1)$ with $y$ being an $\infty$-critical point of $D$.

Remark 3.3. Let $\mathcal{A}:=\left\{C \in \mathcal{K}^{k} \mid \operatorname{as}_{1}(C)=\operatorname{as}_{\infty}(C), k=0,1,2, \ldots\right\}$ be the class of convex bodies whose $p$-measures coincide for all $p$, in all dimensions. Then $\mathcal{A}$ is closed under invertible affine transformations and coproducts of convex bodies, as follows from Theorems 2.6 and 3.2. Observe also that a simplex in $k$ dimensions can be considered as the $(k+1)$-fold coproduct of its vertices (trivially symmetric convex bodies in 0 dimensions). Thus, we have naturally the following questions:

Question 1. Is the class of symmetric convex bodies a generating set for $\mathcal{A}$ under invertible affine transformations and coproducts?

Question 2. Does as $\operatorname{as}_{1}(C)=\operatorname{as}_{\infty}(C)$ hold if $\operatorname{as}_{1}(C)=\operatorname{as}_{2}(C)$ (or, generally, if $\operatorname{as}_{p_{1}}(C)=\operatorname{as}_{p_{2}}(C)$ for distinct $\left.p_{1}, p_{2}\right)$ ?

\section{Acknowledgement}

Our sincere thanks go to the referee for his invaluable comments and suggestions, in particular, for his formulating Question 1, which promotes the value of this paper.

\section{References}

[Asplund et al. 1962] E. Asplund, E. Grosswald, and B. Grünbaum, "On a measure of asymmetry of convex bodies", Proc. Cambridge Philos. Soc. 58 (1962), 217-220. MR 25 \#3425 Zbl 0141.39001 [Besicovitch 1948] A. S. Besicovitch, "Measure of asymmetry of convex curves", J. London Math. Soc. 23 (1948), 237-240. MR 10,320r Zbl 0035.38402 
[Böröczky 2010] K. J. Böröczky, "Stability of the Blaschke-Santaló and the affine isoperimetric inequality”, Adv. Math. 225:4 (2010), 1914-1928. MR 2011j:52016 Zbl 1216.52007

[Chakerian and Stein 1964] G. D. Chakerian and S. K. Stein, "On the symmetry of convex bodies", Bull. Amer. Math. Soc. 70 (1964), 594-595. MR 29 \#517 Zbl 0126.38403

[Dziechcińska-Halamoda and Szwiec 1985] Z. Dziechcińska-Halamoda and W. Szwiec, "On critical sets of convex polyhedra”, Arch. Math. (Basel) 44:5 (1985), 461-466. MR 86j:52003 Zbl 0554.52004

[Eggleston 1952] H. G. Eggleston, "Measure of asymmetry of convex curves of constant width and restricted radii of curvature", Quart. J. Math., Oxford Ser. (2) 3 (1952), 63-72. MR 13,768d Zbl 0046.16003

[Ekström 2000] E. Ekström, "The critical set of a convex body", Project Report 2000:P7, Uppsala University, Department of Mathematics, 2000, available at http://www2.math.uu.se/research/pub/ ekstrom.pdf.

[Gluskin and Litvak 2008] E. D. Gluskin and A. E. Litvak, "Asymmetry of convex polytopes and vertex index of symmetric convex bodies", Discrete Comput. Geom. 40:4 (2008), 528-536. MR 2010d:52025 Zbl 1161.52008

[Groemer 2000] H. Groemer, "Stability theorems for two measures of symmetry", Discrete Comput. Geom. 24:2-3 (2000), 301-311. MR 2001e:52002 Zbl 0960.52005

[Groemer and Wallen 2001] H. Groemer and L. J. Wallen, "A measure of asymmetry for domains of constant width”, Beiträge Algebra Geom. 42:2 (2001), 517-521. MR 2002i:52003 Zbl 0996.52001

[Grünbaum 1963] B. Grünbaum, "Measures of symmetry for convex sets", pp. 233-270 in Proceedings of symposia in pure mathematics (University of Washington, Seattle), vol. VII, edited by V. L. Klee, Amer. Math. Soc., Providence, R.I., 1963. MR 27 \#6187 Zbl 0142.20503

[Guo 2005] Q. Guo, "Stability of the Minkowski measure of asymmetry for convex bodies", Discrete Comput. Geom. 34:2 (2005), 351-362. MR 2006d:52003 Zbl 1079.52003

[Guo 2012] Q. Guo, “On p-measures of asymmetry for convex bodies”, Adv. Geom. 12:2 (2012), 287-301. MR 2911151 Zbl 1242.52004

[Guo and Kaijser 1999] Q. Guo and S. Kaijser, "On the distance between convex bodies", Northeast. Math. J. 15:3 (1999), 323-331. MR 2001g:52007 Zbl 1011.52002

[Guo and Kaijser 2002] Q. Guo and S. Kaijser, "On asymmetry of some convex bodies", Discrete Comput. Geom. 27:2 (2002), 239-247. MR 2002m:52002 Zbl 1010.52003

[Guo and Kaijser 2003] Q. Guo and S. Kaijser, "Approximation of convex bodies by convex bodies", Northeast. Math. J. 19:4 (2003), 323-332. MR 2004k:52006 Zbl 1071.52010

[Hug and Schneider 2007] D. Hug and R. Schneider, "A stability result for a volume ratio", Israel J. Math. 161 (2007), 209-219. MR 2009e:52015 Zbl 1139.52012

[Kaiser 1996] M. J. Kaiser, "The critical point and related symmetry measures of a planar convex set”, Comput. Math. Appl. 32:9 (1996), 79-98. MR 98i:52002 Zbl 0871.52002

[Klee 1953] V. L. Klee, Jr., "The critical set of a convex body”, Amer. J. Math. 75 (1953), 178-188. MR 14,678f Zbl 0050.16604

[Minkowski 1897] H. Minkowski, "Allgemeine Lehrsätze über die convexen Polyeder", Nachr. Ges. Wiss. Göttingen, Math.-Phys. Kl. 1897 (1897), 198-219.

[Mizushima 2000] T. Mizushima, "Estimation of symmetry parameter and tests for symmetry", Math. Japon. 52:3 (2000), 359-376. MR 2002a:62050 Zbl 0965.62032

[Petitjean 2003] M. Petitjean, "Chirality and symmetry measures: a transdisciplinary review", Entropy 5:3 (2003), 271-312. MR 2054025 Zbl 1078.00503 
[Rogers and Shephard 1958] C. A. Rogers and G. C. Shephard, "Some extremal problems for convex bodies", Mathematika 5 (1958), 93-102. MR 21 \#2960 Zbl 0092.15301

[Schneider 1993] R. Schneider, Convex bodies: the Brunn-Minkowski theory, Encyclopedia of Mathematics and its Applications 44, Cambridge University Press, Cambridge, 1993. MR 94d:52007 Zbl 0798.52001

[Schneider 2009] R. Schneider, "Stability for some extremal properties of the simplex", J. Geom. 96:1-2 (2009), 135-148. MR 2011e:52013 Zbl 1201.52008

[Soltan 2005] V. Soltan, "Affine diameters of convex bodies—a survey", Expo. Math. 23:1 (2005), 47-63. MR 2005k:52013 Zbl 1076.52001

[Stein 1956] S. Stein, "The symmetry function in a convex body”, Pacific J. Math. 6 (1956), 145-148. MR 18,228g Zbl 0071.15701

[Toth 2008] G. Toth, "On the structure of convex sets with applications to the moduli of spherical minimal immersions", Beiträge Algebra Geom. 49:2 (2008), 491-515. MR 2010a:53120 Zbl 1167.52008

[Toth 2009] G. Toth, "Asymmetry of convex sets with isolated extreme points", Proc. Amer. Math. Soc. 137:1 (2009), 287-295. MR 2010f:52004 Zbl 1165.52005

[Tuzikov et al. 1997] A. V. Tuzikov, G. L. Margolin, and A. I. Grenov, "Convex set symmetry measurement via Minkowski addition”, J. Math. Imaging Vision 7:1 (1997), 53-68. MR 98a:52004 Zbl 0868.68113

[Tuzikov et al. 2000] A. V. Tuzikov, J. B. Roerdink, and H. J. Heijmans, "Similarity measures for convex polyhedra based on Minkowski addition”, Pattern Recognition 33:6 (2000), 979-995.

[Zouaki 2003] H. Zouaki, "Convex set symmetry measurement using Blaschke addition", Pattern Recognition 36:3 (2003), 753-763. Zbl 1029.68128

Received August 8, 2014. Revised March 7, 2015.

QI GUO

DEPARTMENT OF MATHEMATICS

SUZHOU UNIVERSITY OF SCIENCE AND TECHNOLOGY

SUZHOU, JIANGSU 215009

CHINA

guoqi@mail.usts.edu.cn

JiNFENG GUO

DEPARTMENT OF MATHEMATICS

SUZHOU UNIVERSITY OF SCIENCE AND TECHNOLOGY

SUZHOU, JIANGSU 215009

CHINA

gjfsx@mail.usts.edu.cn

XUNLi SU

DEPARTMENT OF MATHEMATICS

SuZHou University of SCIENCE AND TECHNOLOGY

SuzHOU, JiANGSU 215009 CHINA

hardysoo@163.com 


\title{
PACIFIC JOURNAL OF MATHEMATICS
}

\author{
msp.org/pjm
}

Founded in 1951 by E. F. Beckenbach (1906-1982) and F. Wolf (1904-1989)

\section{EDITORS}

Don Blasius (Managing Editor)

Department of Mathematics

University of California

Los Angeles, CA 90095-1555

blasius@math.ucla.edu

\author{
Paul Balmer \\ Department of Mathematics \\ University of California \\ Los Angeles, CA 90095-1555 \\ balmer@math.ucla.edu \\ Robert Finn \\ Department of Mathematics \\ Stanford University \\ Stanford, CA 94305-2125 \\ finn@math.stanford.edu \\ Sorin Popa \\ Department of Mathematics \\ University of California \\ Los Angeles, CA 90095-1555 \\ popa@math.ucla.edu
}

\author{
Vyjayanthi Chari \\ Department of Mathematics \\ University of California \\ Riverside, CA 92521-0135 \\ chari@math.ucr.edu \\ Kefeng Liu \\ Department of Mathematics \\ University of California \\ Los Angeles, CA 90095-1555 \\ liu@math.ucla.edu \\ Jie Qing \\ Department of Mathematics \\ University of California \\ Santa Cruz, CA 95064 \\ qing@ cats.ucsc.edu
}

\section{PRODUCTION}

Silvio Levy, Scientific Editor, production@msp.org

\section{SUPPORTING INSTITUTIONS}

ACADEMIA SINICA, TAIPEI

CALIFORNIA INST. OF TECHNOLOGY

INST. DE MATEMÁTICA PURA E APLICADA

KEIO UNIVERSITY

MATH. SCIENCES RESEARCH INSTITUTE

NEW MEXICO STATE UNIV.

OREGON STATE UNIV.

\author{
STANFORD UNIVERSITY \\ UNIV. OF BRITISH COLUMBIA \\ UNIV. OF CALIFORNIA, BERKELEY \\ UNIV. OF CALIFORNIA, DAVIS \\ UNIV. OF CALIFORNIA, LOS ANGELES \\ UNIV. OF CALIFORNIA, RIVERSIDE \\ UNIV. OF CALIFORNIA, SAN DIEGO \\ UNIV. OF CALIF., SANTA BARBARA
}

\author{
Daryl Cooper \\ Department of Mathematics \\ University of California \\ Santa Barbara, CA 93106-3080 \\ cooper@math.ucsb.edu \\ Jiang-Hua Lu \\ Department of Mathematics \\ The University of Hong Kong \\ Pokfulam Rd., Hong Kong \\ jhlu@maths.hku.hk \\ Paul Yang \\ Department of Mathematics \\ Princeton University \\ Princeton NJ 08544-1000 \\ yang@math.princeton.edu
}

These supporting institutions contribute to the cost of publication of this Journal, but they are not owners or publishers and have no responsibility for its contents or policies.

See inside back cover or msp.org/pjm for submission instructions.

The subscription price for 2015 is US \$420/year for the electronic version, and \$570/year for print and electronic.

Subscriptions, requests for back issues and changes of subscribers address should be sent to Pacific Journal of Mathematics, P.O. Box 4163, Berkeley, CA 94704-0163, U.S.A. The Pacific Journal of Mathematics is indexed by Mathematical Reviews, Zentralblatt MATH, PASCAL CNRS Index, Referativnyi Zhurnal, Current Mathematical Publications and Web of Knowledge (Science Citation Index).

The Pacific Journal of Mathematics (ISSN 0030-8730) at the University of California, c/o Department of Mathematics, 798 Evans Hall \#3840, Berkeley, CA 94720-3840, is published twelve times a year. Periodical rate postage paid at Berkeley, CA 94704, and additional mailing offices. POSTMASTER: send address changes to Pacific Journal of Mathematics, P.O. Box 4163, Berkeley, CA 94704-0163.

PJM peer review and production are managed by EditFLOW ${ }^{\circledR}$ from Mathematical Sciences Publishers.

\section{PUBLISHED BY}

\section{mathematical sciences publishers \\ nonprofit scientific publishing}

http://msp.org/

(C) 2015 Mathematical Sciences Publishers 


\section{PACIFIC JOURNAL OF MATHEMATICS}

Volume $276 \quad$ No. $2 \quad$ August 2015

Free evolution on algebras with two states, II

257

MiCHAEL ANSHELEVICH

Systems of parameters and holonomicity of A-hypergeometric systems

281

Christine Berkesch Zamaere, STEPHEN GRIFfeth and EZRA Miller

Complex interpolation and twisted twisted Hilbert spaces

287

FÉlix Cabello SÁnChez, Jesús M. F. Castillo and Nigel J. Kalton

The ramification group filtrations of certain function field extensions

309

JEFFREY A. CASTAÑEDA and QINGQUAN WU

A mean field type flow, II: Existence and convergence

JEAN-BAPTISTE CASTÉRAS

Isometric embedding of negatively curved complete surfaces in Lorentz-Minkowski space

BING-LONG CHEN and LE YIN

The complex Monge-Ampère equation on some compact Hermitian manifolds

JIANCHUN CHU

Topological and physical link theory are distinct

ALEXANDER COWARD and JOEL HASS

The measures of asymmetry for coproducts of convex bodies

Qi GuO, JinFEng GuO and XunLi Su

Regularity and analyticity of solutions in a direction for elliptic equations

YongYANG Jin, DONGSHENG LI and XU-JIA WANG

On the density theorem for the subdifferential of convex functions on Hadamard spaces

Mina Movahedi, Daryoush Behmardi and Seyedehsomayeh

HOSSEINI

$L^{p}$ regularity of weighted Szegó projections on the unit disc

SAMANGi MunASINGHE and Yunus E. ZEYTUnCU

Topology of complete Finsler manifolds admitting convex functions

SORIN V. SABAU and KatSUHIRO SHIOHAMA

Variations of the telescope conjecture and Bousfield lattices for localized categories of spectra

F. LUKE WOLCOTT 